SCientia Marina 71(1)

March 2007, 101-113, Barcelona (Spain)

ISSN: 0214-8358

\title{
Antimicrobial activity of Iberian macroalgae
}

\author{
NOEMÍ SALVADOR, AMELIA GÓMEZ GARRETA, LUCA LAVELLI \\ and MARÍA ANTONIA RIBERA \\ Laboratori de Botànica, Facultat de Farmàcia, Universitat de Barcelona, Av. Joan XXIII s/n, 08028 Barcelona, Spain. \\ E-mail: noasalvador@ub.edu
}

\begin{abstract}
SUMMARY: The antibacterial and antifungal activity of 82 marine macroalgae (18 Chlorophyceae, 25 Phaeophyceae and 39 Rhodophyceae) was studied to evaluate their potential for being used as natural preservatives in the cosmetic industry. The bioactivity was analysed from crude extracts of fresh and lyophilised samples against three Gram-positive bacteria, two Gram-negative bacteria and one yeast using the agar diffusion technique. The samples were collected seasonally from Mediterranean and Atlantic coasts of the Iberian Peninsula. Of the macroalgae analysed, 67\% were active against at least one of the six test microorganisms. The highest percentage of active taxa was found in Phaeophyceae (84\%), followed by Rhodophyceae (67\%) and Chlorophyceae (44\%). Nevertheless, red algae had both the highest values and the broadest spectrum of bioactivity. In particular, Bonnemaisonia asparagoides, Bonnemaisonia hamifera, Asparagopsis armata and Falkenbergia rufolanosa (Bonnemaisoniales) were the most active taxa. Bacillus cereus was the most sensitive test microorganism and Pseudomonas aeruginosa was the most resistant. The highest percentages of active taxa from Phaeophyceae and Rhodophyceae were found in autumn, whereas they were found in summer for Chlorophyceae.
\end{abstract}

Keywords: antimicrobial activity, marine macroalgae, Bonnemaisoniales, agar diffusion technique, crude extracts, Iberian Peninsula.

RESUMEN: ACTIVIDAD ANTIMICRobiana DE MACROALGaS MARINAS DE LA Península IBÉRICA. - Se analizó la actividad antibacteriana y antifúngica de 82 macroalgas marinas (18 Chlorophyceae, 25 Phaeophyceae y 39 Rhodophyceae) para valorar su potencial aplicación como conservantes naturales en la industria cosmética. Los extractos crudos de cada taxon, preparados tanto a partir de material fresco como liofilizado, fueron testados frente a tres bacterias Gram positivas, dos bacterias Gram negativas y una levadura, mediante la técnica de difusión en agar. Las muestras fueron recolectadas en diversas localidades de las costas mediterráneas o atlánticas de la Península Ibérica en distintas estaciones del año. El 67\% de todas las macroalgas estudiadas mostraron actividad antimicrobiana frente al menos un microorganismo test de los seis utilizados. El mayor porcentaje de táxones activos lo presentó el grupo de las Phaeophyceae (84\%) seguido por las Rhodophyceae (67\%) y por las Chlorophyceae (44\%). No obstante, las algas rojas fueron las que presentaron el mayor grado de actividad así como el espectro de acción más amplio y, dentro de este grupo, Bonnemaisonia asparagoides, Bonnemaisonia hamifera, Asparagopsis armata y Falkenbergia rufolanosa (Bonnemaisoniales) fueron los táxones más activos. En cuanto a los microorganismos, Bacillus cereus fue el más sensible y Pseudomonas aeruginosa el más resistente. Los tres grupos taxonómicos mostraron una variación estacional en la producción de sustancias antimicrobianas, siendo el otoño la estación con mayor porcentaje de táxones activos para las Phaeophyceae y Rhodophyceae, mientras que para las Chlorophyceae fue el verano.

Palabras clave: actividad antimicrobiana, macroalgas marinas, Bonnemaisoniales, técnica de difusión en agar, extractos crudos, Península Ibérica.

\section{INTRODUCTION}

Several marine organisms produce bioactive metabolites in response to ecological pressures such as competition for space, maintenance of unfouled surfaces, deterrence of predation and the ability to successfully reproduce (König et al., 1994). These bioactive compounds offer rich pharmacological potential (Muñoz, 1992).

There are numerous reports of macroalgae derived compounds that have a broad range of biological activities, such as antibiotic, antiviral, anti- 
neoplastic, antifouling, anti-inflammatory, cytotoxic and antimitotic (Naqvi et al., 1980; Caccamese et al., 1981; Fenical and Paul, 1984; Hodgson, 1984; Ballesteros et al., 1992; Bhosale et al., 2002). Harder (1917) was the first to observe antimicrobial substances secreted by algae. However, it was not until the 1970s that large-scale screening of antimicrobial activity was carried out (Welch, 1962; Glombitza, 1970; Hornsey and Hide, 1974; Henríquez et al., 1977). In the past few decades, macroalgae have been widely recognised as producers of a broad range of bioactive metabolites (Caccamese et al., 1981; Fenical and Paul, 1984; Ma and Tang, 1984; Reichelt and Borowitzka, 1984; Hornsey and Hide, 1985; Rosell and Srivastava, 1987; Febles et al., 1995; Crasta et al., 1997; Melo et al., 1997; Centeno and Ballantine, 1999; Horikawa et al., 1999). However, the results obtained by the aforementioned authors suggest that the production of antimicrobial substances by the same species varies (Pesando, 1990). This intraspecific variability may be due to ecology, the stage of active growth or sexual maturity (Pratt et al., 1951; Chesters and Stott, 1956; Burkholder et al., 1960).

The purpose of this work was to evaluate the antibacterial and antifungal activity of Iberian marine macroalgae. To date, research on biologically active substances of Iberian seaweeds has been scarce (Serarols et al., 1982; Cabañes et al., 1984; Ballesteros et al., 1992). The relationships the geographical zone, sampling season and algal generation have with antimicrobial activity, as well as the influence of sample preparation methods on assay results, are of considerable interest and have scarcely been studied. This information could prove valuable for harvesting algae for industrial applications. In fact, the present study corresponds to the first experimental task of a European project aimed at evaluating using macroalgae as natural preservatives in the cosmetic industry.

\section{MATERIAL AND METHODS}

A total of 82 taxa (18 Chlorophyceae, 25 Phaeophyceae and 39 Rhodophyceae) were sampled at various sites along the northern Mediterranean (Llançà, Port de la Selva, Palamós, Begur, Lloret de Mar, Blanes and the Ebro Delta) and Atlantic (San Sebastián, Guetaria, Ondarreta, Zarauz, Ría de Vigo and Bayona) coasts of Spain. To evaluate the possi- ble influence of sampling season on antimicrobial activity, the maximum possible number of these taxa in each season (winter, spring, summer and autumn) was collected. Seaweeds were collected by scuba diving or snorkelling and preserved on ice until further processing. Seaweed samples were manually cleansed of sand, epiphytes and animals, then rinsed in distilled water to remove salt. Samples from each taxon were prepared using two different treatments: freezing at $-40^{\circ} \mathrm{C}$ (hereafter referred to as fresh) and lyophilisation. The bioactivities of the fresh and lyophilised samples were subsequently compared to determine any differences resulting from the respective preparation methods.

As the bioactivity of Bonnemaisoniales has previously been reported, we carried out complementary studies for some of the taxa present in the Iberian Peninsula (Asparagopsis armata, its tetrasporophyte Falkenbergia rufolanosa, Bonnemaisonia asparagoides and Bonnemaisonia hamifera). The Bonnemaisoniales present on both Atlantic and Mediterranean coasts were collected to assess the relationship between the geographical zone and bioactivity. To evaluate whether antimicrobial activity varies with life-cycle generations of algae, gametophytic and tetrasporophytic generations of $A$. armata were analysed.

The test microorganisms selected were spoiled microorganisms or common human pathogens, and were comprised of the three Gram-positive bacteria Bacillus subtilis (ATCC 6633), Bacillus cereus (identified strain by the CECT) and Staphylococcus aureus (ATCC 29213), the two Gram-negative bacteria Escherichia coli (ATCC 35218) and Pseudomonas aeruginosa (ATCC 9027), and the yeast Candida albicans (ATCC 48867). All cultures were kept on Brain Heart Infusion (BHI) agar plates and stored at $4^{\circ} \mathrm{C}$, except the initial stock cultures, which were stored at $-40^{\circ} \mathrm{C}$ in $\mathrm{BHI}$ broth containing $20 \%$ glycerol.

Solid extracts from fresh and lyophilised material were prepared for all taxa following a modified version of the extraction method of Burkholder et al. (1960). The extracts were obtained by milling algal samples without solvent using a Waring blender and/or manually with a mortar. Due to the high bioactivity observed for the solid extracts of Bonnemaisoniales, we sought to prove this high bioactivity by also obtaining methanolic extracts. The extracts were prepared using a modified version of the method used by Caccamese et al. (1981) from 
algal material (ca. $2 \mathrm{~g}$ of lyophilised or $6 \mathrm{~g}$ of fresh material) homogenised via a Polytron in $10 \mathrm{ml}$ of methanol-toluene (3:1). The extracts were then centrifuged to remove insoluble material, the supernatants were evaporated at reduced pressure, and the solid residue was then dissolved in $1 \mathrm{ml}$ of methanol.

Antimicrobial activity was evaluated by the agar diffusion method, which is the most widely used method for susceptibility testing and is simple, economical and reproducible (Álvarez Benito, 1990). Moreover, this standardised procedure is accepted for determining antimicrobial susceptibility by the National Committee for Clinical Laboratory Standards (NCCLS).

A liquid microorganism suspension corresponding to a $0.5 \mathrm{McF}$ arland scale (standard suspension of barium sulfate which represents $1.5 \times 10^{8}$ bacteri$\mathrm{al} / \mathrm{ml}$ ) was applied to Mueller-Hinton plates using a cotton swab. After a few minutes, to allow complete absorption of the inoculum, the crude extracts were placed on the agar plates. The solid extracts $(0.2 \mathrm{~g})$, obtained from fresh and from lyophilised material of each taxon, were placed in $9.3 \mathrm{~mm}$ diameter wells made on the plates with a sterilised cork borer. The methanolic extracts from Bonnemaisoniales were absorbed onto non-impregnated discs (bioMérieux, $6 \mathrm{~mm}$ diameter), air-dried to eliminate residual solvent, and then placed onto the inoculated plates.

During overnight incubation at $37^{\circ} \mathrm{C}$, the yeast or bacterial lawn grew over the agar surface (Hodgson, 1984), except where it was inhibited by the radial diffusion of antimicrobial compounds of the extracts. The diameter of the inhibition halo is considered to be indicative of the bioactivity of the seaweed extract, and was measured (including the well or disc diameter) with a caliper. Mean diameter values were calculated from triplicate runs of each assay. Standardised values for diameters of the inhibition halo, expressed in $\mathrm{mm}$, produced by the microorganisms against known antibiotics are listed in the literature (Álvarez Benito, 1990). Our results were interpreted according to these values, whereby a diameter less than $1 \mathrm{~mm}$ was interpreted as representing a taxon with trace activity, a diameter between 1 and $20 \mathrm{~mm}$ was interpreted as representing an active taxon, and a diameter larger than 20 $\mathrm{mm}$ was interpreted as representing a taxon with a level of bioactivity sufficient for antibiotic use (hereafter referred to as high activity).

The influence of algal treatment and sampling season on the results from solid extracts was assessed using variance analysis (ANOVA, Statgraphics Plus 5.1, Statistical Graphics Corp., 1994-2001). Both analyses were applied to the most sensitive microorganism, Bacillus cereus, and did not include the taxa belonging to Bonnemaisoniales.

Three analyses of variance were carried out for the Bonnemaisoniales taxa, including in this case all test microorganisms. Bonnemaisoniales from Atlantic and Mediterranean coasts were analysed for their season of maximum activity by two-way ANOVA with geographical zone and test microorganisms as factors. The difference in bioactivity between Mediterranean specimens of A. armata and its tetrasporophyte $F$. rufolanosa was assessed by two-way ANOVA using generations and test microorganisms as factors. To compare the bioactivities of fresh and lyophilised material from Bonnemaisoniales, solid and methanolic extracts were analysed by two-way ANOVA.

\section{RESULTS}

\section{Antimicrobial activity of solid extracts for the whole taxa}

The results for the solid extracts from each season are summarised in Tables 1, 2, 3 and 4. Of the 82 taxa analysed, 55 (67\%) showed antimicrobial activity against at least one test microorganism (Fig. 1). Of these, one Phaeophyceae and five Rhodophyceae showed antimicrobial activity against all six test microorganisms: Hapalospongidion macrocarpum, Asparagopsis armata, its tetrasporophyte Falkenbergia rufolanosa, Osmundea truncata, Plocamium cartilagineum and Rytiphlaea tinctoria (Tables 1-4). The antimicrobial activities of six other red algae (Bonnemaisonia asparagoides, Bonnemaisonia hamifera, Ceramium deslongchampsii, Jania rubens, Peyssonnelia rubra and Wrangelia penicillata) and one brown alga (Cystoseira mediterranea) against five test microorganisms were among the highest (Tables 1-4). However, 18 taxa (22\%) did not show antimicrobial activity against any microorganism assayed (Fig. 1), and nine $(11 \%)$ only showed trace activity against at least one test microorganism (Fig. 1).

This work includes the first descriptions ever published of the bioactivities of 15 of the taxa studied (Tables 1-4). Of these taxa, seven were active against at least one microorganism: the green alga 
TABLE 1. - Antimicrobial activity of solid extracts of Iberian macroalgae in winter. TG= Taxonomic Group (C: Chlorophyceae, P: Phaeophyceae, R: Rhodophyceae); C= Coast (M: Mediterranean, A: Atlantic); Bas= Bacillus subtilis; Bac $=$ B. cereus; Sta= Staphylococcus aureus; $\mathrm{Eco}=$ Escherichia coli $;$ Psa $=$ Pseudomonas aeruginosa $; \mathrm{Can}=$ Candida albicans. Numbers indicate diameter of inhibition halo in mm; $\operatorname{tr}=$ trace $;-=$ inactive.

\begin{tabular}{|c|c|c|c|c|c|c|c|c|c|c|c|}
\hline & & & & & Fresh & & & & Lyopl & nilized & \\
\hline TG C & C Таха & Bas & $\begin{aligned} \text { Gram } \\
\text { Bac }\end{aligned}$ & + Sta & $\begin{array}{l}\text { Gram - } \\
\text { Eco Psa }\end{array}$ & $\begin{array}{c}\text { Yeast } \\
\text { Can }\end{array}$ & Bas & Gram & + & $\begin{array}{l}\text { Gram - } \\
\text { Eco Psa }\end{array}$ & $\begin{array}{r}\text { Yeast } \\
\text { Can }\end{array}$ \\
\hline C $\mathrm{N}$ & M Bryopsis muscosa J.V. Lamouroux & - & - & - & - & - & - & - & - & - & \\
\hline & M Chaetomorpha linum (O.F. Muller) Kützing & - & 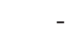 & - & $\operatorname{tr}$ & - & & & & & \\
\hline & M Cladophora rupestris (Linnaeus) Kützing & 11.6 & 11.9 & - & - & $\operatorname{tr}$ & 12.6 & 13.2 & $\operatorname{tr}$ & - & - \\
\hline & M Codium bursa (Linnaeus) C. Agardh & - & - & - & - & - & - & - & - & - & \\
\hline & M Codium coralloides (Kützing) P.C. Silva & - & - & - & - & - & - & - & - & - & - \\
\hline & M Codium vermilara (Olivi) Delle Chiaje & - & & - & - & - & - & - & - & & \\
\hline & M Flabellia petiolata (Turra) Nizamuddin & - & - & - & - & - & - & - & - & - & \\
\hline & M Halimeda tuna (J. Ellis and Solander) J.V. Lamouroux & 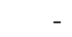 & - & - & - & - & & & & & \\
\hline & M Palmophyllum crassum (Naccari) Rabenhorst & 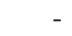 & - & - & - & - & & & & & \\
\hline & M Ulva rigida C. Agardh & - & , & - & - & - & & & & & \\
\hline & M Valonia macrophysa Kützing & - & & - & - & - & & & & & \\
\hline $\mathrm{P} \quad \mathrm{N}$ & M Colpomenia sinuosa (Mertens ex Roth) Derbès and Solier & 13.8 & 14.3 & 14.5 & - & - & 15.4 & 15.8 & 14.8 & - & \\
\hline & M Cystoseira barbata (Stackhouse) C. Agardh . & 10.1 & 11.9 & $\operatorname{tr}$ & - & - & & & & & \\
\hline & $\begin{array}{l}\text { M Cystoseira brachycarpa J. Agardh v. balearica } \\
\text { (Sauvageau) Giaccone }\end{array}$ & & & & - & - & & & & - & \\
\hline & M Cystoseira compressa (Esper) Gerloff and Nizamuddin & 13.4 & 13.4 & 14.1 & - & - & 14.1 & 14.9 & 15.2 & - & \\
\hline & M Cystoseira mediterranea Sauv & $\operatorname{tr}$ & tr & - & - & - & & 15.9 & 12.6 & 13.2 & - \\
\hline & A Cystoseira tamariscifolia $(\mathrm{Hu}$ & 11 & 13 & $\operatorname{tr}$ & - & - & 13.2 & 12.8 & 11.9 & - & \\
\hline & M Dictyopteris polypodioides (A.P. De Candolle) J.V. Lamouro & oux - & & - & - & - & & & & & \\
\hline & M Dictyota dichotoma (Hudson) J.V. Lamouroux var. dichotom & na $\operatorname{tr}$ & 11.6 & $\operatorname{tr}$ & - & - & & & & & \\
\hline & M Dictyota dichotoma (Hudson) J.V. Lamouroux var. intricata & & & & & & & & & & \\
\hline & (C. Agardh) Greville & $\operatorname{tr}$ & 11.6 & - & - & - & & & & & \\
\hline & M Dictyota spiralis Montagne & 12.8 & 11.8 & $\operatorname{tr}$ & - & - & 11.6 & 11.7 & $\operatorname{tr}$ & - & - \\
\hline & $\begin{array}{l}\text { M Hapalospongidion macrocarpum (Feldmann) León Álvarez } \\
\text { \& González González }\end{array}$ & & & & & & & & & & \\
\hline & (Linnaeus) J.V. Lamouroux & 13.5 & 12.4 & 11.3 & $-\quad-$ & - & 13 & 13.5 & 14.1 & _ & \\
\hline & M Scytosiphon lomentari & 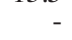 & 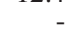 & 10 & - & - & & & & & \\
\hline & M Stypocaulon & - & - & - & - & - & & & & & \\
\hline & M Taonia atome & & 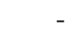 & & - & - & - & - & $\operatorname{tr}$ & & 12.3 \\
\hline & M Zanardinia typus (Nardo) P.C. Silva & 16.5 & 13.7 & 16.8 & - & - & - & $\operatorname{tr}$ & - & -12.9 & $\operatorname{tr}$ \\
\hline $\mathrm{R} N$ & M Ast & 29.4 & 30.2 & 22.2 & 20.825 .5 & 32 & 38.9 & 51.1 & 35.1 & 39.927 .3 & 53.2 \\
\hline & M Ba & & $\operatorname{tr}$ & & - & 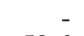 & & 20.6 & $\operatorname{tr}$ & - & \\
\hline & A Bonnema & 46.8 & 34 & 40.5 & 12.5 & 52.6 & 52.1 & 56 & 41.4 & 15.4 & 37.1 \\
\hline & M Borne & - & - & & - & & & & & & \\
\hline & M Ceramium ciliatum (J. Ellis) Ducluz & & $\operatorname{tr}$ & $\operatorname{tr}$ & $\operatorname{tr}$ & 15 & - & - & - & - & - \\
\hline & M Ceramium deslongchampsii (Chauvin) ex Duby & 13.9 & 14.9 & 16.5 & 13.714 .1 & - & & & & & \\
\hline & M Corallin & 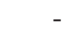 & 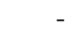 & - & $\operatorname{tr}$ & - & - & - & - & - & 14.7 \\
\hline & A Falkenbergia rufolanosa (Harvey) Schmitz & & & & 18.2 & & 18 & 26.1 & 18.6 & 17.916 .9 & 26.3 \\
\hline & M Falkenberg & 31.7 & 32.3 & 24.1 & 26.123 .6 & 43.6 & 20.3 & 31.4 & 23.2 & 24.220 .8 & 43.3 \\
\hline & M Gastroclonium clava & - & & & - & 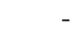 & & & & & \\
\hline & in) P.C. Silva & - & & & - & & - & - & & - & \\
\hline & M Gracilar & - & 19.5 & 11.5 & 18.6 & 27.3 & - & - & - & - & \\
\hline & M Grateloupia filici & - & & - & - & 12.9 & & & & & \\
\hline & M Jar & $\operatorname{tr}$ & tr & & - & 13.4 & - & & 12.8 & - & 15.1 \\
\hline & M Laurencia intricata J.V. Lamouroux & $\operatorname{tr}$ & 11.6 & 13.9 & $\operatorname{tr}$ & 12.4 & - & $\operatorname{tr}$ & 15 & 1212.7 & $\operatorname{tr}$ \\
\hline & V. Lamouroux & 15.1 & & 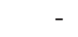 & $\operatorname{tr}$ & - & & & & & \\
\hline & M Nemal & & & & & & & & & & \\
\hline & ng) K.W. Nam and Maggs & 18.7 & 21.4 & 17.2 & 12.714 .1 & 15.3 & 21.4 & 26.8 & 15.8 & 1916.1 & 27.1 \\
\hline & M Peyssonnelia rubra $(\mathrm{G} 1$ & 10.7 & 11.8 & 14.1 & -12.4 & 15.1 & & & & & \\
\hline & M Plocamium cartilagine & 11 & 12.4 & 12 & -10.8 & 13.8 & & & & & \\
\hline & M Porphy & & & & $\operatorname{tr}$ & 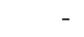 & & & & & \\
\hline & M Porphyra linearis Greville & - & & & - & - & & & & & \\
\hline & M Pterocladiella capillacea (S.G. Gmelin) Santelices & & & & & & & & & & \\
\hline & & $\operatorname{tr}$ & $\operatorname{tr}$ & - & - & & & - & & - & \\
\hline & M Rissoella verruculosa (A. Bertoloni) J. Agardh & - & & & - & - & - & - & - & - & \\
\hline & M Rytiphlaea tinctoria (Clemente) C. Ágardh & 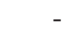 & 11.9 & 10.7 & - & - & 15.6 & 16.2 & 16.3 & 11.6 & \\
\hline & M Schottera nicaeensis (J.V. Lamouroux ex Duby) & & & & & & & & & & \\
\hline & Guiry and Hollenberg & & & & - & & & & & & \\
\hline & M Sphaerococcus coronopifolius Stackhouse & 12.1 & 14.3 & 12.7 & $\operatorname{tr}$ & 18.6 & - & - & - & - & $-\quad 15.5$ \\
\hline
\end{tabular}

Enteromorpha muscoides, the brown algae Fucus spiralis var. platycarpus and Spatoglossum solieri and the red algae Boergeseniella fruticulosa, Gracilaria dura, R. tinctoria, Schottera nicaeensis and Scinaia furcellata. G. dura and R. tinctoria were notably active, the former against yeast and Escherichia coli, 
TABLE 2. - Antimicrobial activity of solid extracts of Iberian macroalgae in spring. TG= Taxonomic Group (C: Chlorophyceae, P: Phaeophyceae, R: Rhodophyceae); C = Coast (M: Mediterranean, A: Atlantic); Bas= Bacillus subtilis; Bac= B. cereus; $\mathrm{Sta}=$ Staphylococcus aureus; $\mathrm{Eco}=$ Escherichia coli $; \mathrm{Psa}=$ Pseudomonas aeruginosa $; \mathrm{Can}=$ Candida albicans. Numbers indicate diameter of inhibition halo in $\mathrm{mm}$; $\operatorname{tr}=$ trace; - = inactive.

\begin{tabular}{|c|c|c|c|c|c|c|c|c|c|c|c|c|}
\hline & & & & & & & & & & Lyo & hilized & \\
\hline & & & & Gram & & Gram & Yeast & & Gram & & Gram & Yeast \\
\hline TG & $\mathrm{C}$ & Taxa & Bas & $\mathrm{Bac}$ & Sta & Eco Psa & Can & Bas & Bac & Sta & Eco Psa & Can \\
\hline $\mathrm{C}$ & & Bryopsis muscosa J.V. Lamouroux & - & 12.2 & $\operatorname{tr}$ & - & - & $\operatorname{tr}$ & 23 & 27.1 & - & - \\
\hline & & $\begin{array}{l}\text { Cladophora rupestris (Linnaeus) Kützing } \\
\text { Codium fragile (Suringar) Hariot subsn }\end{array}$ & 14 & 13.4 & 13.4 & - & $\operatorname{tr}$ & & & & & \\
\hline & & tomentosoides (Goor) P.C. Silva & - & - & - & - & - & & & & & - \\
\hline & M & Enteromorpha intestinalis (Linnaeus) Nees & - & - & - & - & - & & & & & \\
\hline & M & Flabellia petiolata (Turra) Nizamuddin & - & & - & - & - & - & & - & - & - \\
\hline & $\mathrm{M}$ & $\begin{array}{l}\text { Halimeda tuna (J. Ellis and Solander) } \\
\text { J.V. Lamouroux }\end{array}$ & - & - & - & - & - & & & & & \\
\hline & M & Ulva rigida $\mathrm{C}$. Agardh & - & $\operatorname{tr}$ & 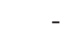 & - & 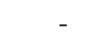 & - & - & - & - & - \\
\hline & $\mathrm{M}$ & Valonia macrophysa Kützing & - & - & - & - & - & & & & & \\
\hline $\mathrm{P}$ & & Bifurcaria bifurcata R. Ross & $\operatorname{tr}$ & $\operatorname{tr}$ & 10.5 & - & - & 12.3 & 13 & 16.4 & - & - \\
\hline & & (Lightfoot) Prud'homme van Reine & - & - & - & - & - & - & - & 14.1 & - & - \\
\hline & & Colpomenia sinuosa (Mertens ex Roth) Derbès & & & & & & & & & & \\
\hline & & $\begin{array}{l}\text { and Solier } \\
\text { Cystoseira barbata (Stackhouse) C. Agar }\end{array}$ & 12.4 & 10.8 & 13.7 & - & - & 13.7 & 13.7 & 15.7 & - & - \\
\hline & & Cystoseira brachycarpa J. Agardh v. balearica & & & & & & & & & - & \\
\hline & & (Sauvageau) Giaccone & $\operatorname{tr}$ & 11 & 10.4 & - & - & - & - & - & - & - \\
\hline & M & I Cystoseira compressa (Esper) Gerloff and & 129 & 128 & 142 & & & & & & & \\
\hline & M & Cystoseira mediterranea Sauvageau & 10.8 & 12.8 & 14.2 & 106 & - & 13.5 & 13.6 & 135 & $\operatorname{tr}_{116}$ & - \\
\hline & A & Cystoseira tamariscifolia (Hudson) Papenfuss & 11.5 & 12.3 & 12 & 10.0 & - & 13.4 & 14.4 & 19.7 & 12.7 & - \\
\hline & & Dictyopteris polypodioides (A.P. De Candolle) & & & & & & & & & & \\
\hline & & J.V.Lamouroux & - & - & - & - & - & & & & & \\
\hline & M & Dictyota dichotoma (Hudson) J.V. Lamouroux & & & & & & & & & & \\
\hline & & var. intricata (C. Agardh) Greville & 12.9 & 13.1 & 10.9 & - & 12.8 & 12.8 & 12 & 11.6 & - & $\operatorname{tr}$ \\
\hline & M & I Dictyota spiralis Montagne & & 11.4 & $\operatorname{tr}$ & - & - & & & $\operatorname{tr}$ & - & \\
\hline & A & Fucus spiralis Linnaeus var. platycarpus Batters & 10.8 & 11 & 11.6 & - & - & 12.6 & 12.2 & 13.3 & - & - \\
\hline & $\begin{array}{l}\mathrm{M} \\
\mathrm{M}\end{array}$ & $\begin{array}{l}\text { Padina pavonica (Linnaeus) J.V. Lamouroux } \\
\text { Phyllariopsis brevipes (C. Agardh) E.C. }\end{array}$ & 12.6 & 11.9 & 13.1 & - & - & & & & & \\
\hline & & Henry and South & - & - & - & - & - & & & & & \\
\hline & M & Scytosiphon lomentaria (Lyngbye) Link & - & - & $\operatorname{tr}$ & - & - & & & & & \\
\hline & $\mathrm{M}$ & [ Spatoglossum solieri (Chauvin ex Montagne) & & & & & & & & & & \\
\hline & & Kützi & 12.8 & 10.7 & 11 & - & - & 11.2 & 11.6 & 11.4 & - & - \\
\hline & M & I Sporochnus pedunculatus (Hudson) C. Agardh & 12.1 & 15.3 & 10.9 & - & 15 & - & $\operatorname{tr}$ & & - & 14.5 \\
\hline & M & I Stypocaulon scoparium (Linnaeus) Kützing & - & $\operatorname{tr}$ & - & - & - & - & - & 12.4 & - & \\
\hline & $\mathrm{M}$ & I Taonia atomaria (Woodward) J. Ágardh & 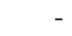 & & 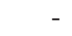 & - & - & $\operatorname{tr}$ & 22.8 & 20.5 & - & 14.5 \\
\hline $\mathrm{R}$ & M & I Asparagopsis armata Harvey & 29.6 & 26.2 & 21.2 & 22.1 & 33.5 & & & & & \\
\hline & A & Asparagopsis armata Harvey & 25.4 & 28.8 & 19.9 & 14 & 24.4 & 37.7 & 47.1 & 29.8 & 19.6 & 42.7 \\
\hline & M & [ Boergeseniella fruticulosa (Wulfen) Kylin & 11.7 & 12.2 & 10.2 & - & 13.4 & & & & & \\
\hline & M & $\begin{array}{l}\text { Bonnemaisonia asparagoides } \\
\text { (Woodward) C. Agardh }\end{array}$ & 55.2 & 82.9 & 68.5 & 18.1 & 49.7 & 31.5 & 41.2 & 27.3 & 18.9 & 33.5 \\
\hline & A & Bonnemaisonia asparagoides & & & & & & & & & & \\
\hline & A & $\begin{array}{l}\text { (Woodward) C. Agardh } \\
\text { Bonnemaisonia hamifera Hariot }\end{array}$ & 68.3 & 64.9 & 65.4 & 18.7 & 59.8 & 69.9 & 78.3 & 70.5 & 22.7 & 68.2 \\
\hline & M & Callithamnion granulatum (Ducluzeau) C. Agardh & 10.2 & 11.1 & 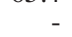 & 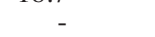 & 11.7 & & & & & \\
\hline & & Ceramium ciliatum (J. Ellis) Ducluzeau & & & & & & - & 11.4 & - & - & 12.5 \\
\hline & M & I Ceramium rubrum auctorum & - & 10.8 & - & - & 11.1 & & & & & \\
\hline & M & I Corallina elongata $\mathrm{J}$. Ellis and Solander & 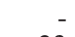 & & & 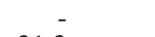 & 14.3 & & & & & \\
\hline & M & I Falkenbergia rufolanosa (Harvey) Schmitz & 23 & 23.7 & 21.2 & 21.3 & 23.9 & 20.5 & 14 & 18.3 & 19.1 & 30.6 \\
\hline & M & I Gastroclonium clavatum (Roth) Ardisson & - & $\operatorname{tr}$ & & - & - & - & - & - & - & - \\
\hline & M & $m$ (S.G. Gmelin) P.C. Silva & - & & & - & & - & - & - & - & - \\
\hline & M & I Gracilaria dura (C. Agardh) J. Agardh & - & 19.7 & 11.8 & 19.7 & 25.5 & - & - & - & - & - \\
\hline & M & I Grateloupia filicina (J.V. Lamouroux) C. Agardh & - & - & & - & & - & - & - & - & - \\
\hline & M & I Jania rubens (Linnaeus) J.V. Lamourou & - & & & - & 14.8 & & & & & \\
\hline & M & I Laurencia obtusa (Hudson) J.V. Lamouroux & - & - & 10.6 & - & 10.5 & & & & & \\
\hline & M & I Liagora viscida (Forsskal) C. Agardh & - & & 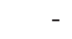 & - & - & - & - & - & - & - \\
\hline & M & I Nemalion helminthoides (Velley) Batters & & - & 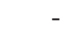 & - & - & - & - & - & - & - \\
\hline & M & I Osmundea truncata (Kützing) & 156 & 17 & 115 & 127 & 217 & 152 & 136 & 134 & $\operatorname{tr}$ & 127 \\
\hline & M & I Pterocladiella capillacea (S.G. Gmelin) & 15.0 & $1 / .2$ & $11 . J$ & 12.1 & 21.1 & & 15.0 & 15.4 & tr & 12.1 \\
\hline & & Santelices and Hommersand & - & & & & & - & - & - & - & - \\
\hline & M & I Rytiphlaea tinctoria (Clemente) C. Agardh & 15.8 & 16.3 & 19.6 & 11.4 & 15.2 & & & & & \\
\hline & & (J.V. Lamouroux ex Duby) & & - & & & & & & & & \\
\hline & M & $\begin{array}{l}\text { Sulry and Hollenberg } \\
\text { I Sphaerococcus coronopifolius Stackhouse }\end{array}$ & 12.1 & 15.3 & 10.9 & - & 15 & - & $\operatorname{tr}$ & - & - & 14.5 \\
\hline
\end{tabular}


TABLE 3. - Antimicrobial activity of solid extracts of Iberian macroalgae in summer. TG= Taxonomic Group (C: Chlorophyceae, P: Phaeophyceae, R: Rhodophyceae); C= Coast (M: Mediterranean, A: Atlantic); Bas= Bacillus subtilis; Bac= B. cereus; Sta= Staphylococcus aureus; $\mathrm{Eco}=$ Escherichia coli $;$ Psa $=$ Pseudomonas aeruginosa $; \mathrm{Can}=$ Candida albicans. Numbers indicate diameter of inhibition halo in mm; $\operatorname{tr}=$ trace; - = inactive.

\begin{tabular}{|c|c|c|c|c|c|c|c|c|c|}
\hline & & & & & Fresh & & & philized & \\
\hline TG & C Taxa & Bas & $\begin{array}{l}\mathrm{Sram}- \\
\mathrm{Bac}\end{array}$ & Sta & $\begin{array}{l}\text { Gram - } \\
\text { Eco Psa }\end{array}$ & $\begin{array}{l}\text { Yeast } \\
\text { Can }\end{array}$ & $\begin{array}{c}\text { Gram + } \\
\text { Bas Bac Sta }\end{array}$ & $\begin{array}{l}\text { Gram - } \\
\text { Eco Psa }\end{array}$ & $\begin{array}{l}\text { Yeast } \\
\text { Can }\end{array}$ \\
\hline $\mathrm{C}$ & M Acetabularia acetabulum (Linnaeus) P.C.Silva & 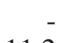 & 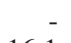 & - & - & - & 7 & - & \\
\hline & M Bryopsis corymbosa J. Agardh & 11.2 & 16.1 & $\operatorname{tr}$ & - & $\operatorname{tr}$ & 13.717 .812 .7 & $\operatorname{tr}$ & 13.5 \\
\hline & M Cladophora lehmannianna (Linderberg) Kützing & - & - & - & - & - & & & \\
\hline & M Codium bursa (Linnaeus) C. Agardh & - & - & - & - & - & 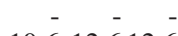 & - & - \\
\hline & M Codium coralloides (Kützing) P.C. Silva & - & - & - & - & - & 10.612 .612 .6 & - & - \\
\hline & A Codium tomentosum Stackhouse & - & & - & - & - & & & \\
\hline & M Codium vermilara (Olivi) Delle Chiaje & & & & & & $\operatorname{tr} 11.911 .9$ & - & 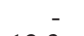 \\
\hline & M Flabellia petiolata (Turra) Nizamuddin & - & 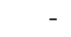 & 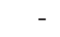 & - & - & - - & - & 12.3 \\
\hline & M Halimeda tuna (J. Ellis and Solander) J.V. Lamouroux & - & - & - & - & - & - & - & $\operatorname{tr}$ \\
\hline & M Palmophyllum crassum (Naccari) Rabenhorst & 12.4 & 11.8 & 12.1 & 13 & - & & & \\
\hline & M Ulva rigida C. Agardh & - & & - & - & - & - & - & - \\
\hline & M Valonia macrophysa Kützing & - & & & & & & & \\
\hline $\mathrm{P}$ & $\begin{array}{l}\text { A Bifurcaria bifurcata R. Ross } \\
\text { M Cladostephus spongiosum f. verticillatum (Lightfoot) }\end{array}$ & $\operatorname{tr}$ & _- & $\operatorname{tr}$ & - & $\operatorname{tr}$ & & & \\
\hline & Prud'homme van Reine & - & $\operatorname{tr}$ & - & - & - & - & - & - \\
\hline & M Colpomenia sinuosa (Mertens ex Roth) Derbès and Solier & 15.3 & 15.9 & 17.1 & - & - & 1414.815 .4 & $\operatorname{tr}$ & - \\
\hline & M Cystoseira barbata (Stackhouse) C. Agardh & 12.1 & 12.1 & 12.5 & - & - & $11.812 .4 \quad 18$ & - & - \\
\hline & M Cystoseira brachycarpa J. Agardh v. balearica & & & & & & & & \\
\hline & M Cystoseira compressa (Esper) Gerloff and Nizamuddin & 10.3 & 11.7 & 11.8 & - & - & 11.812 .816 .2 & - & - \\
\hline & M Cystoseira mediterranea Sauvageau & 12.1 & 12 & 12.2 & - & - & 1414.315 .4 & $\operatorname{tr}$ & - \\
\hline & M Dictyopteris polypodioides (A.P. De Candolle) & & & & & & & & \\
\hline & J.V. Lamouroux & - & & - & - & - & - & - & - \\
\hline & M Dictyota dichotoma (Hudson) J.V. Lamouroux var. dichotom & $a-$ & $\operatorname{tr}$ & - & - & - & - 1112.3 & - & - \\
\hline & $\begin{array}{l}\text { M Dictyota dichotoma (Hudson) J.V. Lamouroux var. } \\
\text { intricata (C. Agardh) Greville }\end{array}$ & $\operatorname{tr}$ & $\operatorname{tr}$ & - & - & - & - & - & - \\
\hline & M Hapalospongidion macrocarpum (Feldmann) & & & & & & & & \\
\hline & León Álvarez \& González González & 18.7 & 19.3 & 17.2 & 13 & 12.3 & & & \\
\hline & A Laminaria ochroleuca La Pylaie & - & - & - & - & - & & & \\
\hline & M Padina pavonica (Linnaeus) J.V. Lamouroux & $\operatorname{tr}$ & $\operatorname{tr}$ & $\operatorname{tr}$ & - & - & -12.4 & - & - \\
\hline & M Stypocaulon scoparium (Linnaeus) Kützing & - & - & - & - & - & & & \\
\hline & M Taonia atomaria (Woodward) J. Agardh & - & - & - & - & - & - & - & - \\
\hline & M Zanardinia typus (Nardo) P.C. Silva & 17.3 & 16.2 & 16.7 & - & - & 13.515 .216 .3 & - & - \\
\hline $\mathrm{R}$ & M Callithamnion granulatum (Ducluzeau) C. Agardh & - & 12.2 & 10.5 & - & $\operatorname{tr}$ & & & \\
\hline & M Ceramium ciliatum (J. Ellis) Ducl & - & & & - & 11.9 & -12.111 .7 & - & $\operatorname{tr}$ \\
\hline & M Corallina elongata J. Ellis and Solander & $\operatorname{tr}$ & $\operatorname{tr}$ & - & $\operatorname{tr}$ & 12.7 & $\operatorname{tr} 10.513 .2$ & 11 & 13.7 \\
\hline & M Falkenbergia rufolanosa (Harvey) Schmitz & 17.6 & 17.8 & 19.4 & 14 & 20.8 & & & \\
\hline & A Gelidium corneum (Hudson) J.V. Lamouroux & - & - & - & - & - & & & \\
\hline & M Gelidium spinosum (S.G. Gmelin) P.C. Silva & - & - & - & - & $\sigma$ & - & - & - \\
\hline & M Grateloupia filicina (J.V. Lamouroux) C. Agardh & & 13.9 & $\operatorname{tr}$ & - & 10.5 & & & \\
\hline & M Gymnogongrus crenulatus (Turner) J. Agardh & 13.8 & 17.4 & $\operatorname{tr}$ & - & 15.3 & & & \\
\hline & M Halymenia floresia (Clemente) C. Agardh & $\operatorname{tr}$ & & $\operatorname{tr}$ & $\operatorname{tr}$ & - & & & \\
\hline & M Hypnea musciformis (Wulfen) J.V. Lamouroux & & & & & & -14.3 & - & \\
\hline & M Jania rubens (Linnaeus) J.V. Lamouroux & 11.2 & 12.2 & 11.4 & 10.9 & 14 & -11.4 & - & 15.1 \\
\hline & M Laurencia intricata J.V. Lamouroux & 10.4 & 12.8 & $\operatorname{tr}$ & - & $\operatorname{tr}$ & $\operatorname{tr} 11.511 .1$ & - & - \\
\hline & M Laurencia obtusa (Hudson) J.V. Lamouroux & 17.9 & 19.3 & 17.3 & - & $\operatorname{tr}$ & 15.719 .417 .1 & - & $\operatorname{tr}$ \\
\hline & M Liagora tetrasporifera Boergesen & - & - & - & - & - & & & \\
\hline & M Liagora viscida (Forsskal) C. Agardh & - & & 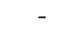 & - & - & - & - & - \\
\hline & M Nemalion helminthoides (Velley) Batters & & & & - & & - & 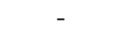 & - \\
\hline & $\begin{array}{l}\text { M Plocamium cartilagineum (Linnaeus) P.S. Dixon } \\
\text { M Pterocladiella capillacea (S.G. Gmelin) }\end{array}$ & 15.1 & 16.7 & 14 & 10.6 & 14.2 & 12.614 .814 .2 & 13.1 & $\operatorname{tr}$ \\
\hline & Santelices and Hommersand & $\operatorname{tr}$ & 11 & $\operatorname{tr}$ & $\operatorname{tr}$ & 10.8 & - & - & - \\
\hline & M Rissoella verruculosa (A. Bertoloni) J. Agardh & - & & - & - & - & & & \\
\hline & (J.V. Lamouroux ex Duby) & & & & & & & & \\
\hline & Guiry and Hollenberg & $\operatorname{tr}$ & $\operatorname{tr}$ & - & - & $\operatorname{tr}$ & & & \\
\hline & M Scinaia complanata (Collins) Cotton & - & 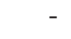 & - & - & - & $\operatorname{tr}$ & - & - \\
\hline & M Scinaia furcellata (Turner) J. Agardh & - & - & 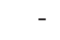 & - & - & & & \\
\hline & M Sphaerococcus coronopifolius Stackhouse & - & - & - & - & - & - & - & - \\
\hline & M Wrangelia penicillata (C. Agardh) C. Agardh & 11.2 & 13.1 & 12 & 10.6 & 14.1 & & & \\
\hline
\end{tabular}

and the latter primarily against the Gram-positive bacteria. The remaining seven taxa, Chlorophyceae Acetabularia acetabulum and Cladophora lehmanni- anna, Phaeophyceae Laminaria ochroleuca and Rhodophyceae Bornetia secundiflora, Gelidium corneum, Liagora tetrasporifera and Scinaia com- 
TABLE 4. - Antimicrobial activity of solid extracts of Iberian macroalgae in autumn. TG= Taxonomic Group (C: Chlorophyceae, P: Phaeophyceae, R: Rhodophyceae); C= Coast (M: Mediterranean, A: Atlantic); Bas= Bacillus subtilis; Bac= B. cereus; $\mathrm{Sta}=$ Staphylococcus aureus; $\mathrm{Eco}=$ Escherichia coli $; \mathrm{Psa}=$ Pseudomonas aeruginosa $; \mathrm{Can}=$ Candida albicans. Numbers indicate diameter of inhibition halo in $\mathrm{mm}$; $\operatorname{tr}=$ trace $;-=$ inactive

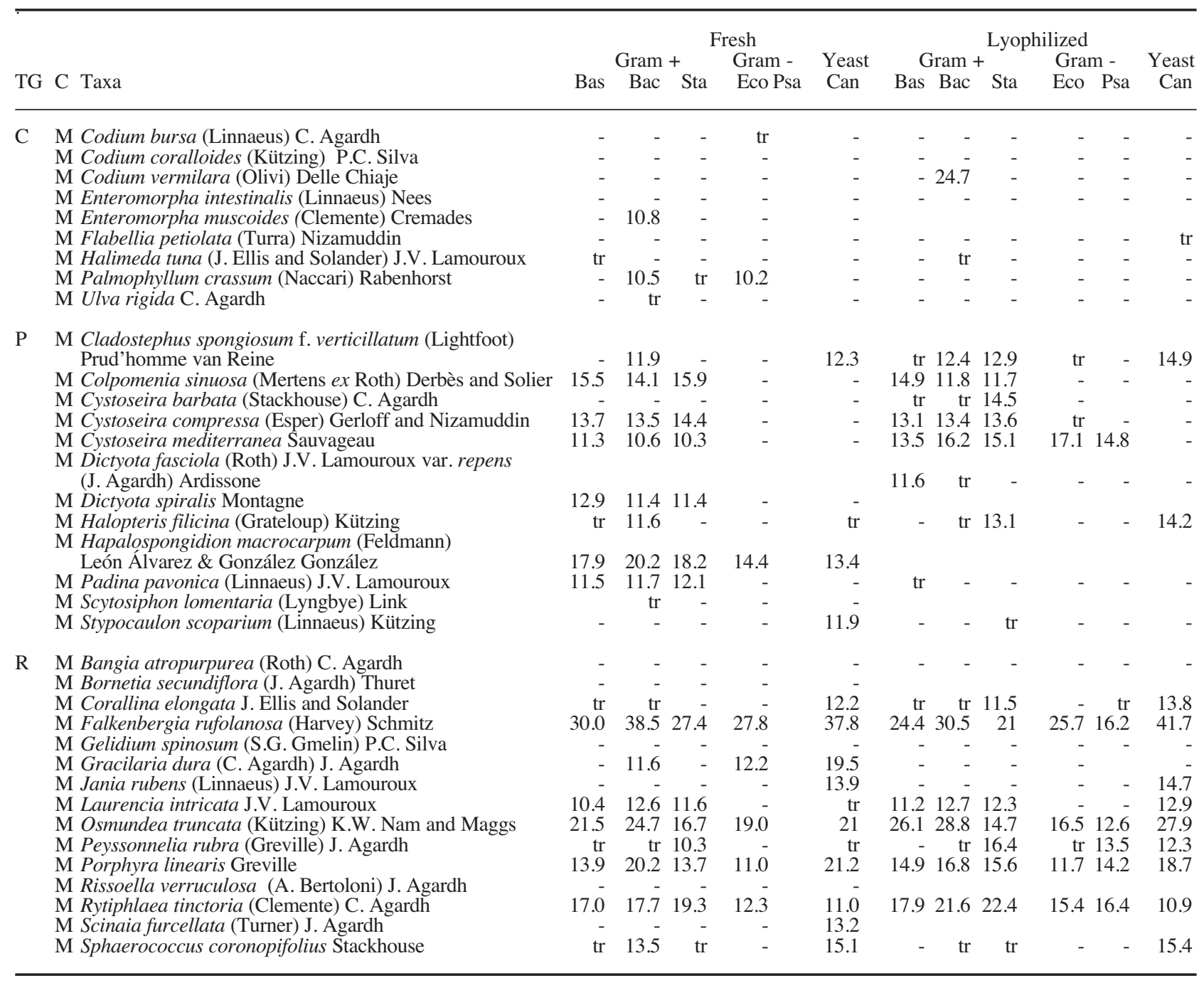

planata, did not demonstrate any activity against the test microorganisms.

The sensitivity of the test microorganisms was in the following decreasing order: Bacillus cereus (inhibited by $57 \%$ of tested taxa), Staphylococcus aureus (55\%), Candida albicans (44\%), Bacillus subtilis (43\%), E. coli (22\%) and Pseudomonas aeruginosa $(21 \%)$.

\section{Antimicrobial activity of solid extracts according to taxonomic group}

Chlorophyceae had the lowest percentage of active taxa (44\%, Fig. 1), with low bioactivity and a narrow spectrum of action that was generally limited to Gram-positive bacteria. Nevertheless, two taxa
Active 目Traces $\square$ Inactive

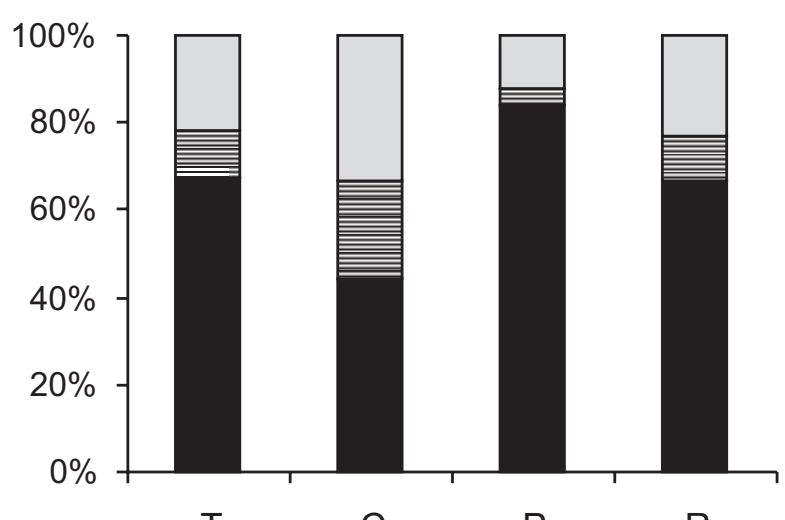

FIG. 1. - Percentage of inactive, trace active, active taxa (solid extracts). T, total taxa; C, Chlorophyceae; P, Phaeophyceae; R, Rhodophyceae. 


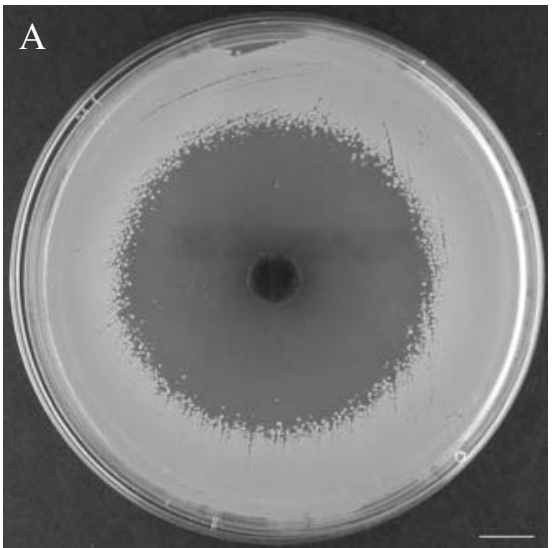

FIG. 2. - Bonnemaisonia asparagoides bioassays (solid extracts). A, against Baci

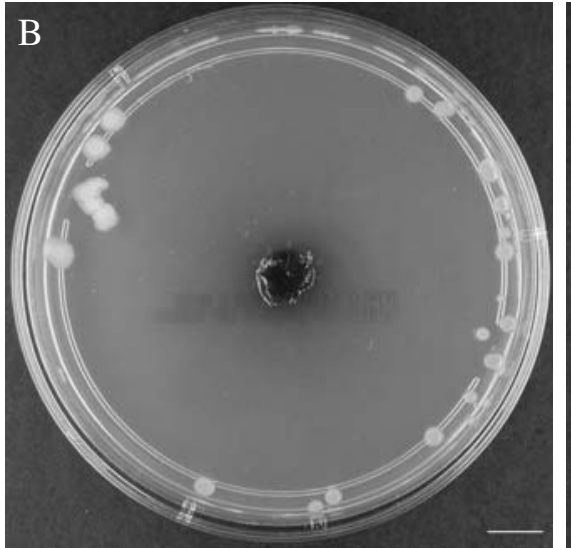

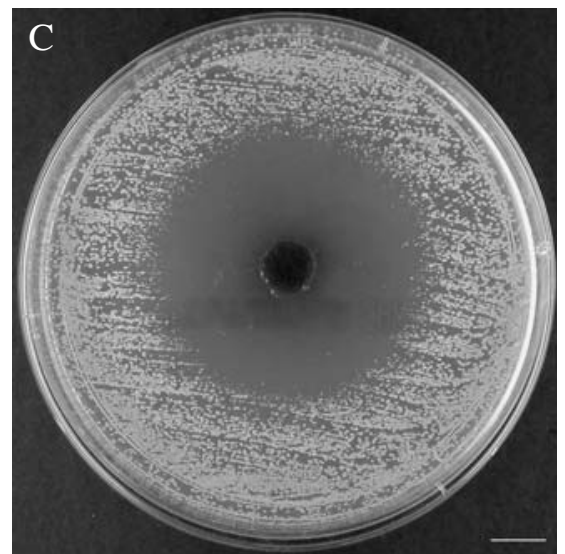

$\mathrm{C}$, against Candida albicans. Scale bar $=10 \mathrm{~mm}$. were active against yeast, and Palmophyllum crassum was the only Chlorophyceae active against Escherichia coli (Tables 3-4). The largest number of active Chlorophyceae taxa belonged to the genus Bryopsis, with Bryopsis muscosa and B. corymbosa showing the highest values and the broadest spectrum respectively.

Phaeophyceae had the highest percentage of active taxa (84\%, Fig. 1), although these did not exhibit the highest antimicrobial activity among the taxa tested. The action spectrum of Phaeophyceae was broader than that of Chlorophyceae, as some taxa were active against the yeast or the Gram-negative bacteria (Tables 1-4). The highest activities for Phaeophyceae were observed for taxa from the genera Cystoseira, Dictyota and Taonia. Although all of these genera were active against the Gram-positive bacteria, Cystoseira was also active against the Gram-negative bacteria whereas Dictyota and Taonia showed antifungal action. Finally, among all of the brown algae tested, Hapalospongidion macrocarpum showed the highest antimicrobial activity.

Rhodophyceae demonstrated the highest antimicrobial activity and the highest number of taxa

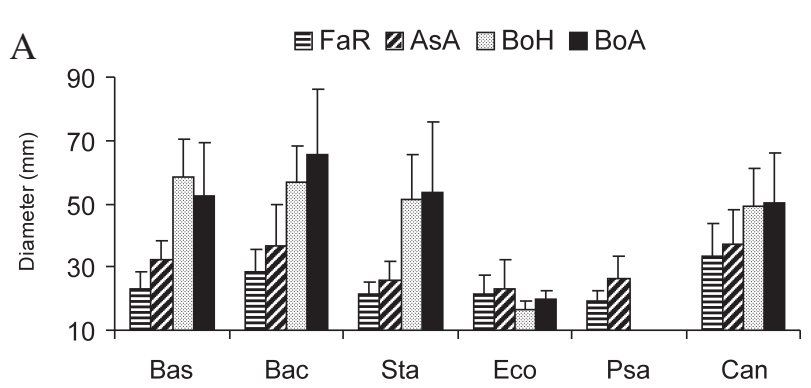

active against Gram-negative bacteria and yeast, thus it was the group with the broadest spectrum of action. However, the percentage of active Rhodophyceae (67\%) was lower than that of Phaeophyceae (Fig. 1). Within this group, Ceramiales and Gigartinales had noteworthy antimicrobial activity, and Bonnemaisoniales was the order that had the highest bioactivity (Fig. 2).

\section{Antimicrobial activity of the Bonnemaisoniales}

Both solid and methanolic extracts from B. hamifera and $B$. asparagoides of the genus Bonnemaisonia showed a broad spectrum and high bioactivity; although the values for inhibition obtained from methanolic extracts were lower than those from solid extracts (Fig. 3). The microorganisms most inhibited by these taxa were the Grampositive bacteria: Bacillus subtilis for B. hamifera, and Bacillus cereus and Staphylococcus aureus for B. asparagoides (Tables 1,2).

The solid and methanolic extracts of Asparagopsis armata and its tetrasporophyte Falkenbergia rufolanosa also exhibited a broad spectrum and high activity, although as above, methanolic extracts were

B

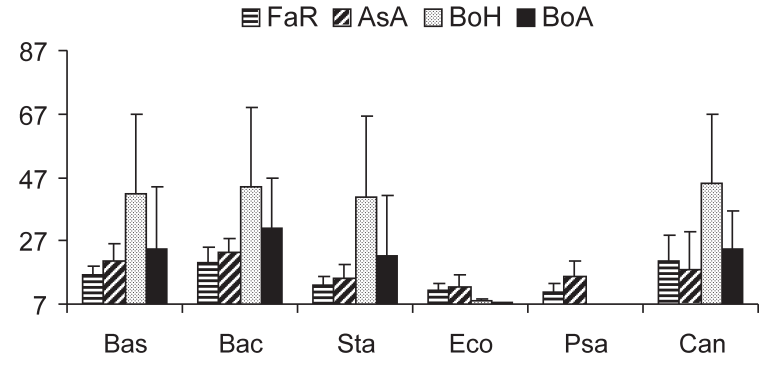

FIG. 3. - Average diameter $( \pm \mathrm{SE})$ of inhibition halo of Falkenbergia rufolanosa (FaR), Asparagopsis armata (AsA), Bonnemaisonia hamifera $(\mathrm{BoH})$, Bonnemaisonia asparagoides $(\mathrm{BoA})$ against each test microorganism. BaS, Bacillus subtilis; Bac, B. cereus; Sta, Staphylococcus aureus; Eco, Escherichia coli; Psa, Pseudomonas aeruginosa; Can, Candida albicans. A, solid extracts; B, methanolic extracts. 
somewhat less active than solid extracts (Fig. 3). For both generations the most inhibited microorganism was Candida albicans (Tables 1-4). The variation in antimicrobial activity between the algal generations was assessed by means of two-way ANOVA, using the test microorganisms and the generations as factors. Significant differences were observed for the generation $\left(\mathrm{F}_{1,54}=8.89, \mathrm{p}<0.01\right)$, A. armata was more active than $F$. rufolanosa, and for the microorganisms $\left(\mathrm{F}_{5,54}=10.29, \mathrm{p}<0.001\right), C$. albicans was the most sensitive. The interaction term was not significant $\left(F_{5,54}=0.79, p>0.05\right)$, which indicates that the action spectrum did not vary with the generation. Three average groups were obtained in relation to the Tukey comparisons: one group comprised of $C$. albicans (the most inhibited microorganism), another group with all the Gram-positive bacteria and E. coli and finally one made up of the most resistant microorganism $P$. aeruginosa.

Out of all tested taxa, the Bonnemaisoniales had the maximum activity against all microorganisms: $A$. armata showed the maximum bioactivity against Gram-negative bacteria whereas the Bonnemaisonia species presented the maximum bioactivity against Gram-positive bacteria and yeast (Fig. 3).

\section{Effects of sample preparation on antimicrobial activity}

For each season, the antimicrobial activity between taxa and different algal treatment (fresh and lyophilised material) was compared by two-way ANOVA. No significant differences between treatments were found (winter: $\mathrm{F}_{1,28}=1.23, \mathrm{p}>0.05$; spring: $\mathrm{F}_{1,49}=1.85, \mathrm{p}>0.05$; summer: $\mathrm{F}_{1,41}=2.30$, $p>0.05$; autumn: $F_{1,35}=2.79, p>0.05$ ), although the mean values of the lyophilised material were higher than those of the fresh material for all seasons except autumn. In contrast, significant differences among taxa were detected (winter: $\mathrm{F}_{14,28}=11.78$, $\mathrm{p}<0.001$; spring: $\mathrm{F}_{17.49}=7.60, \mathrm{p}<0.001$; summer: $\mathrm{F}_{11,41}=26.03, \mathrm{p}<0.001$; autumn: $\mathrm{F}_{15,35}=14.31$, $\mathrm{p}<0.001)$. Furthermore, the interaction term was significant in all seasons except autumn (winter: $\mathrm{F}_{14,28}$ $=4.52, \mathrm{p}<0.05$; spring: $\mathrm{F}_{17,49}=11.5$, $\mathrm{p}<0.001$; summer: $\mathrm{F}_{11,41}=2.64, \mathrm{p}<0.05$; autumn: $\mathrm{F}_{15,35}=1.38$, $\mathrm{p}>0.05$ ), which indicates that effects of sample preparation on observed activities varied with taxa. Nevertheless, the bioactivity observed for lyophilised samples suggests that lyophilisation may provide better extraction of compounds.
For the order Bonnemaisoniales, the two treatments (fresh and lyophilised) were compared across taxa by two-way ANOVA for solid and for methanolic extracts. The differences between treatments were significant for methanolic extracts $\left(F_{1,173}\right.$ $=55.91, \mathrm{p}<0.001)$, but not for solid extracts $\left(\mathrm{F}_{1,255}\right.$ $=0.1, \mathrm{p}>0.05)$. Significant differences between species were found for both solid $\left(\mathrm{F}_{3,255}=39.62\right.$, $\mathrm{p}<0.001)$ and methanolic extracts $\left(\mathrm{F}_{3,173}=13.5\right.$, $\mathrm{p}<0.001)$. The interaction term was also significant for both solid $\left(\mathrm{F}_{3,255}=9.03, \mathrm{p}<0.001\right)$ and methanolic extracts $\left(\mathrm{F}_{3,173}=18.7, \mathrm{p}<0.001\right)$. For Asparagopsis armata, lyophilisation was the most effective treatment for both types of extracts, whereas for its tetrasporophyte Falkenbergia rufolanosa, similar results were obtained from fresh and lyophilised material. For Bonnemaisonia species, the results varied according to the extracts: solid extracts from fresh samples had higher bioactivities than those from lyophilised samples, whereas the results were the opposite for the methanolic extracts.

\section{Seasonal variation of antimicrobial activity}

Autumn and spring were the seasons with the highest percentage of active taxa against at least one test microorganism (69\% and $67 \%$ respectively), followed by winter (56\%) and summer (50\%) (Fig. 4). At a taxonomic group level, for Phaeophyceae and Rhodophyceae the highest percentage of active taxa was also in autumn; however, for Chlorophyceae it was in summer (Fig. 4). In contrast, bioactivity was not significantly different between seasons for any group (one-way ANOVA):

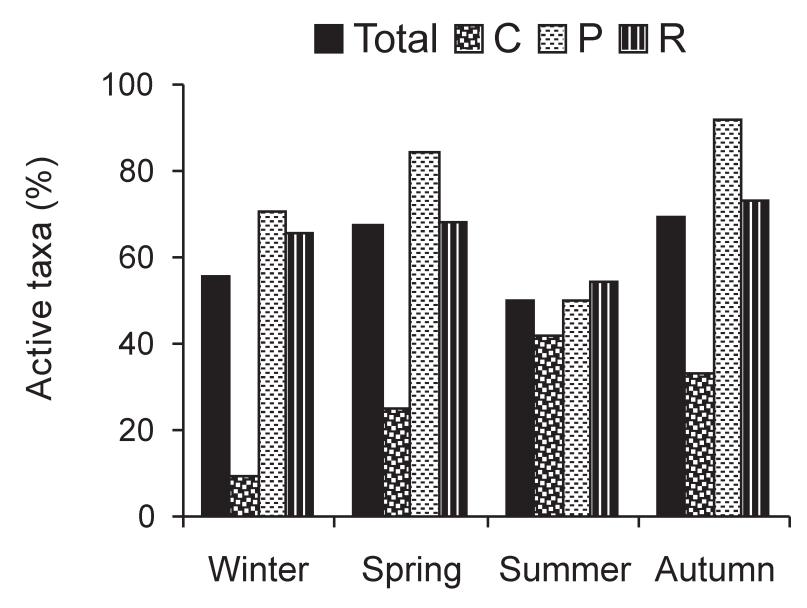

FIG. 4. - Seasonal variation of the percentage of active taxa (solid extracts). T, total taxa; C, Chlorophyceae; P, Phaeophyceae; R, Rhodophyceae. 
TABLE 5. - Taxa for which a broader spectrum of action was observed compared to previous studies on non-Mediterranean and Mediterranean samples. Bas= Bacillus subtilis; Bac= B. cereus; Sta= Staphylococcus aureus; Eco= Escherichia coli; Psa= Pseudomonas aeruginosa; Can= Candida albicans.

non-Mediterranean samples

Asparagopsis armata (Bac, Psa, Can)

Bangia atropurpurea (Sta, Can)

Callithamnion granulatum (Bas, Bac, Sta, Can)

Ceramium ciliatum (Bac, Sta, Can)

Cladophora rupestris (Bas, Bac, Sta)

Codium coralloides (Bac, Sta)

Codium vermilara (Bas, Bac)

Corallina elongata (Bas, Bac, Eco, Psa)

Halopteris filicina (Bas, Bac, Sta)

Hapalospongidion macrocarpum (Bac, Can)

Jania rubens (Bac, Eco)

Peyssonnelia rubra (Bas, Bac, Sta, Eco, Psa, Can)

Plocamium cartilagineum (Bac)

Sphaerococcus coronopifolius (Bac, Sta, Eco)

Taonia atomaria (Bac, Sta, Can)
Mediterranean samples

Bangia atropurpurea (Sta, Eco)

Bryopsis corymbosa (Bas, Bac, Sta, Eco, Can)

Codium vermilara $($ Sta)

Falkenbergia rufolanosa (Bac)

Jania rubens (Eco)

Plocamium cartilagineum (Psa, Can)

Wrangelia penicillata (Bas, Psa)
Chlorophyceae $\left(\mathrm{F}_{3,33}=2.51, \mathrm{p}>0.05\right)$, Phaeophyceae $\left(\mathrm{F}_{3,162}=1.29, \mathrm{p}>0.05\right)$, Rhodophyceae $\left(\mathrm{F}_{3,134}=2.55, \mathrm{p}>0.05\right)$.

The bioactivity values of the taxa were evaluated by two-way ANOVA with taxonomic group and season as factors. No significant differences between seasons $\left(\mathrm{F}_{3,330}=0.68, \mathrm{p}>0.05\right)$ were found, whilst they were found among taxonomic groups $\left(\mathrm{F}_{2,330}=9.63, \mathrm{p}<0.05\right)$. The significant interaction term found $\left(\mathrm{F}_{6,330}=3.32, \mathrm{p}<0.05\right)$ reflected the different variations in bioactivity of the taxonomic groups according to season: Chlorophyceae and Rhodophyceae demonstrated maximum activity during spring and autumn-winter respectively, and Phaeophyceae had constant activity throughout the year.

The greatest antimicrobial activity observed for the order Bonnemaisoniales against all test microorganisms was in winter for Asparagopsis armata and in spring for Bonnemaisonia hamifera (Tables 1-4). However, it must be taken into account that these taxa were only available during the winter-spring period due to their own seasonal dynamic. Falkenbergia rufolanosa, which was present all year-round, showed the greatest activity in autumn-winter.

\section{Geographical variation of antimicrobial activity}

Since the species Asparagopsis armata, Falkenbergia rufolanosa and Bonnemaisonia asparagoides were available from both Atlantic and Mediterranean coasts, their bioactivities were evaluated as a function of geographical zone by two-way ANOVA. The Mediterranean populations of $A$. armata and $F$. rufolanosa were significantly more active than those from the Atlantic $\left(\mathrm{F}_{1,20}=15.65\right.$, $\mathrm{p}<0.05 ; \mathrm{F}_{1,24}=31.64, \mathrm{p}<0.001$ respectively), whereas the opposite trend was observed for $B$. asparagoides $\left(\mathrm{F}_{1,20}=31.44, \mathrm{p}<0.001\right)$. Significant differences among the microorganisms assayed were found for the three taxa (A. armata: $\mathrm{F}_{4,20}=$ $18.31, \mathrm{p}<0.001 ; \mathrm{F}$. rufolanosa: $\mathrm{F}_{5,24}=20.31$, $\mathrm{p}<0.001 ;$ B. asparagoides: $\left.\mathrm{F}_{4,20}=55.21, \mathrm{p}<0.001\right)$. The analysis of the interaction terms showed that the differences were always significant for the three Bonnemaisoniales (A. armata: $\mathrm{F}_{4,20}=4.58, \mathrm{p}<0.05$; F. rufolanosa: $\mathrm{F}_{5,24}=3.43, \mathrm{p}<0.05 ;$ B. asparagoides: $\left.\mathrm{F}_{4,20}=15.62, \mathrm{p}<0.001\right)$, therefore the bioactivity of each species against each microorganism varied with their geographic location.

\section{DISCUSSION AND CONCLUSIONS}

The antimicrobial activities of several of the algae assayed differed from those previously reported. An extended spectrum of action was observed for sixteen taxa compared to studies performed with non-Mediterranean (Baker, 1984; Espeche et al., 1984; Reichelt and Borowitzka, 1984; Usmanghani et al., 1984; Hornsey and Hide, 1985; Ballantine et al., 1987; Navarro et al., 1990; Padmakumar and Ayyakkannu, 1997) as well as Mediterranean samples (Caccamese et al., 1980, 1981, 1985; Serarols et al., 1982; Moreau et al., 1984; Ballesteros et al., 1992) (Table 5). Dictyopteris polypodioides, Halimeda tuna and Hypnea musciformis, three taxa reported as active in other surveys of nonMediterranean samples (Hornsey and Hide, 1974; Sreenivasa Rao and Parekh, 1981; Usmanghani et 
al., 1984; Campos-Takaki et al., 1988; Navarro et al., 1990; Pérez et al., 1990; Padmakumar and Ayyakkannu, 1997) and Mediterranean samples (Ballesteros et al., 1992), were inactive in our study. Finally, Padina pavonica, reported as an inactive taxa in previous Indian (Padmakumar and Ayyakkannu, 1997) and Mediterranean studies (Khaleafa et al., 1975; Ballesteros et al., 1992), showed antibiotic activity for the first time in our work, namely against Bacillus subtilis, B. cereus and Staphylococcus aureus.

The aforementioned observations and the differences in bioactivity between Mediterranean and Atlantic specimens of the Bonnemaisoniales observed in this work suggest that the bioactivity of the same taxon can vary with the geographical sampling zone. Martí et al. (2004) pointed out that these differences could depend on ecological parameters such as irradiance and nutrients.

Our observations of the effects of the sample preparation method/algal treatment (i.e., fresh or lyophilised) on bioactivity revealed that lyophilisation generally allows greater compound extraction. However, as the differences with the fresh material were not significant, it was not possible to determine the most universally efficient treatment. Previous studies that compared different treatments are scarce and were carried out on only a few taxa. Campos-Takaki et al. (1988) and Padmini Sreenivasa Rao et al. (1986) compared fresh and dried algal material; their results also showed lower activity in extracts from fresh tissue than in extracts from dried material. This is probably due to a higher dilution of the bioactive metabolites in the fresh material because of the higher water content. Only Della Pietà et al. (1996) employed lyophilised material, among other materials, but they did not compare their results. Nevertheless, we can conclude from their results that, as in our study, the lyophilised material showed the highest values of antimicrobial activity.

As regards seasonal variation of bioactivity, for all of the taxa tested, autumn was the season with the highest percentage of active taxa against at least one test microorganism, followed by spring. These results agree with those obtained from Indian samples by Sreenivasa Rao and Parekh (1981), and Arun Kumar and Rengasamy (2000), and from Mediterranean samples by Martí et al. (2004). In contrast, in the study carried out by Hornsey and Hide (1974) using Atlantic samples, the most active season was spring. In relation to taxonomic groups, the season with the highest percentage of active taxa was autumn for Phaeophyceae and Rhodophyceae, and summer for Chlorophyceae. However, the results observed for Chlorophyceae did not concur with the constant production of active compounds by this group throughout the year reported by Padmakumar and Ayyakkannu (1997). Some authors have associated peak activity with physiological phenomena; however, the peaks observed in the present work could not be attributed to a single biological process. In some taxa, such as Bonnemaisonia hamifera and Falkenbergia rufolanosa, the peak of bioactivity observed in our study may be related to the reproductive or growth period, as reported by some authors (Hornsey and Hide, 1974; Moreau et al., 1984; Muñoz, 1992). However, in other cases, such as for Osmundea truncata, the peak of bioactivity (autumn-winter) occurred after the reproductive period (springsummer). This finding was in agreement with that of Martí et al. (2004), who stated that peak bioactivity may be related to processes of ageing and allocation of resources from growth or reproduction to production of toxic compounds.

Of all the Iberian taxa screened, the highest antimicrobial activity was observed for Rhodophyceae, among which the order Bonnemaisoniales was the most active. Among the taxa tested in the present work, Bonnemaisonia asparagoides and $B$. hamifera had the highest degree of antimicrobial action against Gram-positive bacteria and yeast. Likewise, A. armata, $F$. rufolanosa, and its tetrasporophyte, which have been highlighted by other authors previously (Serarols et al., 1982; Cabañes et al., 1984; Pesando and Caram, 1984; Ballesteros et al., 1992), presented the highest activity against Gramnegative bacteria out of all the taxa tested in the present article. Comparing both generations, the gametophyte exhibited a broader spectrum and higher degree of antimicrobial action than its tetrasporophyte. Literature data about differences in antimicrobial activity between generations of the same species are scarce. In contrast to our results, Hornsey and Hide (1985) reported higher activity for the tetrasporophyte (Trailliella intrica$t a)$ than for the gametophyte (B. hamifera).

Preservatives are described as substances that guarantee microbiologically safe products. After 
perfumes, preservatives are the cosmetic ingredients that cause the most skin irritations, allergies and atopic reactions. Based on the results of this paper, we suggest that the taxa B. asparagoides, $B$. hamifera and $F$. rufolanosa may have potential as industrial preservatives, analogous to the currently used A. armata (Seguin et al., 1995; Algues et Mer 2002). Out of these, F. rufolanosa could be the most suitable taxon for use as a natural preservative due to its year-round presence and its easiness to culture. Nevertheless, due to the high bioactivity obtained against Gram-positive bacteria and yeast, for the two Bonnemaisonia species, and against Gram-negative bacteria for $F$. rufolanosa, we propose a mixture of their active extracts to obtain a preservative with a broad spectrum of action. Moreover, these taxa merit further studies both with the aim of isolating their active metabolites and for assaying culture methods for supplying algal biomass for industry. We suggest analysing other taxa of this order for which antimicrobial activity is unknown but probably notable, such as $B$. clavata, which has never been tested before because it is generally misidentified (Salvador et al., 2006) with B. asparagoides.

\section{ACKNOWLEDGEMENTS}

The authors thank Dr. J. Rull and Mr. A. Manghisi (University of Barcelona) for their help in the field work and their comments; Dr. F. Cinelli and his research group (University of Pisa) for their collaboration in the beginning of this study and their valuable suggestions; Dr. S. Senesi (University of Pisa) for providing the microorganism cultures; Dr. A. Marqués (University of Barcelona) for providing the Pseudomonas aeruginosa culture and for her helpful comments; Dr. C. Casares and Dr. J. A. Seoane Camba (University of Barcelona) for providing Atlantic samples; Mr. J. A. Navas, Mr. R. Bou, Mr. M. Mumbrú and Dr. C. Codina and his research group (University of Barcelona) for their technical assistance in obtaining methanolic extracts; the Drug Development Service at the University of Barcelona for lyophilisation of the samples; and Mrs A. Henderson and Mr. Gregory Y. Qushair for reviewing the English text.

This study was supported by the project NATURE (CRAFT-2001-70571).

\section{REFERENCES}

Algues et Mer SARL. - 2002. Method of obtaining an antibacterial and/or antifungal extract from the algae, Bonnemaisoniaceae. Inventor: J-Y. Moigne. Off. gaz. U.S. Pat. Trademark Off. Pat., invention patent, US 6346252B1.

Álvarez Benito, Ma. V. - 1990. Manual de técnicas en microbiología clínica. Asociación Española de Farmacéuticos Analistas, San Sebastian.

Arun Kumar, K. and R. Rengasamy. - 2000. Evaluation of antibacterial potential of seaweeds occurring along the coast of Tamil Nadu, India against the plant pathogenic bacterium Xanthomonas oryzae pv. oryzae (Ishiyama) Dye. Bot. Mar., 43: $409-415$.

Baker, J.T. - 1984. Seaweeds in pharmaceutical studies and applications. Hydrobiologia 116/117: 29-40.

Ballantine, D.L., W.H. Gerwick, S.M. Velez, E. Alexander and P. Guevara. - 1987. Antibiotic activity of lipid-soluble extracts from Caribbean marine algae. Hydrobiologia, 151/152: 463-469.

Ballesteros, E., D. Martín and M.J. Uriz. - 1992. Biological activity of extracts from some Mediterranean macrophytes. Bot. Mar., 35: 481-485.

Bhosale, S.H., V.L. Nagle and T.G. Jagtap. - 2002. Antifouling potential of some marine organisms from India species of Bacillus and Pseudomonas. Mar. Biotechnol., 4: 111-118.

Burkholder, P.R., L.M. Burkholder and L.R. Almodóvar. - 1960. Antibiotic activity of some marine algae of Puerto Rico. Bot. Mar., 2: 149-156.

Cabañes, F.J., L. Abarca, M.A. Calvo and J.A. Seoane. - 1984. Determinación de la capacidad antifúngica de algas mediterráneas. $2^{a}$ Reunión conjunta de micología. Resúmenes de ponencias y comunicaciones, p.171.

Caccamese, S., R. Azzolina, G. Furnari, M. Cormaci and S. Grasso. - 1980. Antimicrobial and antiviral activities of extracts from Mediterranean algae. Bot. Mar., 23: 285-288.

Caccamese, S., R. Azzolina, G. Furnari, M. Cormaci and S. Grasso. - 1981. Antimicrobial and antiviral activities of some marine algae from eastern Sicily. Bot. Mar., 24: 365-367.

Caccamese, S., R.M. Toscano, G. Furnari and M. Cormaci. - 1985. Antimicrobial activities of red and brown algae from southern Italy coast. Bot. Mar., 28: 505-507.

Campos-Takaki, G.M., M.B.S. Diu, M.L. Koening and E.C. Pereira. - 1988. Screening of marine algae from Brazilian northeastern coast for antimicrobial activity. Bot. Mar., 31: 375-377.

Centeno, P.O.R. and D.L. Ballantine. - 1999. Effects of culture conditions on production of antibiotically active metabolites by the marine alga Spyridia filamentosa. J. Appl. Phycol., 10: 453-460.

Chesters, C.G. and J.A. Stott. - 1956. Production of antibiotic substances by seaweeds. Proc. Int. Seaweed Symp., 2: 49-54.

Crasta, P.J., N.S. Raviraja and K.R. Sridhar. - 1997. Antimicrobial activity of some marine algae of southwest coast of India. Indian J. Mar. Sci., 26: 201-205.

Della Pietà, F., L. Panizzi, P. L Cioni, F. Cinelli and I. Morelli. 1996. Attività antimicrobica di estratti di Caulerpa taxifolia su batteri e micetti terrestri. In: M.A. Ribera, E. Ballesteros, C.F. Boudouresque, A. Gómez and V. Gravez (eds.). Second International Workshop on Caulerpa taxifolia, pp. 261-264. Publ. Univ. Barcelona, Barcelona.

Espeche, M.E., E.R. Fraile and A.M.S. Mayer. - 1984. Screening of Argentine marine algae for antimicrobial activity. Hydrobiologia, 116/117: 525-528.

Febles, C.I., A. Arias, A. Hardisson, A. Sierra López and M.C. GilRodríguez. - 1995. Antimicrobial activity of extracts from some Canary species of Phaeophyta and Chlorophyta. Phytotherapy Res., 9: 385-387.

Fenical, W. and V.J. Paul. - 1984. Antibiotic and cytotoxic terpenoids from tropical green algae of the family Udoteaceae. Hidrobiologia, 116/117: 137-140.

Glombitza, K.W. - 1970. Antimicrobial constituents in algae. Quantitative determination of acrylic acid in sea-algae. Planta Med., 18: 210-221.

Harder, R. - 1917. Ernährungsphysiologische Untersuchungen an Cyanophyceen, hauptsächlich am endophytischen Nostoc punctiforme. Z. Bot. 9: 145.

Henríquez, P., R. Zemelman, M.A. Moncada and I.L. Benoit. - 
1977. Propiedades antibióticas de algas marinas. Bol. Soc. Biol. Concepción, 11: 119-122.

Hodgson, L.M. - 1984. Antimicrobial and antineoplastic activity in some south Florida seaweeds. Bot. Mar., 27: 387-390.

Horikawa, M., T. Noro and Y. Kamel. - 1999. In vitro anti-methicillin-resistant Staphylococcus aureus. Activity found in extracts of marine algae indigenous to the coastline of Japan. $J$. Antibiot., 52: 186-189.

Hornsey, I.S. and D. Hide. -1974 . The production of antimicrobial compounds by British marine algae. I. Antibiotic-producing marine algae. Br. Phycol. J., 9: 353-361.

Hornsey, I.S. and D. Hide. -1985 . The production of antimicrobial compounds by British marine algae. IV. Variation of antimicrobial activity with algal generation. Br. Phycol. J., 20: 21-25.

Khaleafa, A.F., M.A.M. Kharboush, A. Metwalli, A.F. Mohsen and A. Serwi.- 1975. Antibiotic (fungicidal) action from extracts of some seaweeds. Bot. Mar., 18: 163-165.

König, G.M., A.D. Wright, O. Stiche, C.K. Angerhofer and J.M. Pezzuto. - 1994. Biological activities of selected marine natural products. Planta Med., 60: 532-537.

Ma, J.W. and W.C. Tang. - 1984. Screening for antimicrobial activities in marine algae from the Qingdao coast, China. Hydrobiologia, 116/117: 517-520.

Martí, R., M.J. Uriz and X. Turon. - 2004. Seasonal and spatial variation of species toxicity in Mediterranean seaweed communities: correlation to biotic and abiotic factors. Mar. Ecol. Prog. Ser., 282: 73-85.

Melo, V.M.M., D.A. Medeiros, F.J.B. Rios, L.I.M. Castelar and A. de F.F.U. Carvalho. -1997. Antifungal properties of proteins (agglutinins) from the red alga Hypnea musciformis (Wulfen) Lamouroux. Bot. Mar., 40: 281-284.

Moreau, J., D. Pesando, and B. Caram. - 1984. Antifungal and antibacterial screening of Dictyotales from the French Mediterranean coast. Hydrobiologia, 116/117: 521-524.

Muñoz, A. - 1992. Drogas del mar. Sustancias biomédicas de algas marinas. Servicio de Publicaciones e Intercambio Científico, Universidad de Santiago de Compostela, Santiago de Compostela.

Naqvi, S.W.A., S.Y. Kamat, L. Fernandes, C.V.G. Reddy. - 1980. Screening of some marine plants from the Indian coast for biological activity. Bot. Mar., 24: 51-55.

Navarro, J.N., A. Rodríguez, N. Vásquez, A. Sánchez and M.A. Rivera. - 1990. Efectos antibióticos de las algas marinas de Puerto Rico. Rev. Fac. Ocean. Pesq. Cs. Alimentarias, 2: 165-173.

Padmakumar, K. and K. Ayyakkannu. - 1997. Seasonal variation of antibacterial and antifungal activities of the extracts of marine algae from southern coasts of India. Bot. Mar., 40: 507-515.

Padmini Sreenivasa Rao, P., P. Sreenivasa Rao and S.M. Karmarkar. - 1986. Antibacterial substances from brown algae. II. Efficiency of solvents in the evaluation of antibacterial substances from Sargassum johnstonii Setchell et Gardner. Bot. Mar., 29: 503-507.

Pérez, G.R.M., A.J.G. Avila, G.S. Pérez, C.A. Martínez and C.G. Martínez. - 1990. Antimicrobial activity of some American algae. J. Ethnopharmacol., 29: 111-116.

Pesando, D. - 1990. Antibacterial and antifungal activities of marine algae. In: I. Akatsuka (ed.), Introduction to Applied Phycology, pp. 3-26. SPB Academic Publishing, The Hague.

Pesando, D. and B. Caram. - 1984. Screening of marine algae from the French Mediterranean coast for antibacterial and antifungal activity. Bot. Mar., 27: 381-386.

Pratt, R., R.H. Mautner, G.M. Gardner, Y. Sha and F. Dufrenoy.1951. Report on the antibiotic activity of seaweed extracts. $J$. Amer. Pharm. Assoc. Sci. Edn., 40: 575-579.

Reichelt, J.L. and M.A. Borowitzka. - 1984. Antimicrobial activity from marine algae: results of a large-scale screening programme. Hydrobiologia, 116/117: 158-168.

Rosell, K.G. and L.M. Srivastava. - 1987. Fatty acids as antimicrobial substances in brown algae. Hydrobiologia, 151/152: 471-475.

Salvador Soler, N., A. Gómez Garreta, and M.A. Ribera Siguan. 2006. Mapas de distribución de algas marinas de la Península Ibérica y las islas Baleares. Bonnemaisonia (Bonnemaisoniaceae, Rhodophyta). Bot. Complut., 30: 159-164.

Seguin, M.C., A. Franco, E. Feno, J.Y. Moigne and F. Bresdin. 1995. Extraction de composés organiques de silicium biologiquement actifs d'origine algale. Brevet $\mathrm{n}^{\circ}$ FR 2732022 .

Serarols, M.D., M.C. Hernández and J.A. Seoane. -1982 . Sobre la actividad antibiótica de ciertas especies de algas del Mediterráneo. Collect. Bot. (Barcelona), 13: 919-927.

Sreenivasa Rao, P. and K.S. Parekh. - 1981. Antibacterial activity of Indian seaweed extracts. Bot. Mar., 24: 577-582.

Usmanghani, K., M. Shameel, M. Sualeh, K.H. Khan and Z.A. Mahmood. - 1984. Antibacterial and antifungal activities of marine algae from Karachi seashore of Pakistan. Fitoterapia, 55: 73-77

Welch, A.M. - 1962. Preliminary survey fungistatic properties of marine algae. J. Bacteriol., 83: 97-99.

Received June 16, 2006. Accepted July 21, 2006.

Scient. ed.: I. Uriz.

Published online February 26, 2007. 
